\title{
Demographic evaluation of children with SFU grades hydronephrosis
}

\author{
Nazli Gulriz Ceri
}

Assistant Professor, Department of Anatomy, Faculty of Medicine, Adnan Menderes University, Aydin, TURKEY. Email: drnazligulrizceri@gmail.com

\begin{abstract}
Background: Nonspecific symptoms in renal diseases make diagnosing difficult. The aim of the postnatal observations, other than diagnosis, is to identify cases likely to benefit from early surgery. We investigated the degrees and prevalence of hydronephrosis in patients referred to our hospital's children nephrology policlinic. Materials and Methods: This research was carried out with renal USG taken from 1919 patients between 0-15 years of age. Patients were also evaluated in terms of demographic parameters and hydronephrosis We classified postnatal hydronephrosis according to SFU staging and evaluated demographic parameters. Results: G1 hydronephrosis was observed in 159 cases (74 girls and 85 boys); G2 hydronephrosis in 33 cases ( 9 girls and 24 boys); G3 hydronephrosis in 18 cases ( 7 girls and 11 boys); and G4 hydronephrosis in 10 cases (4 girls and 6 boys) on the right side. Whereas, G1 hydronephrosis was observed in 191 cases ( 73 girls and 118 boys), G2 hydronephrosis in 55 cases (14 girls and 41 boys), G3 hydronephrosis in 36 cases (6 girls and 30 boys) and G4 hydronephrosis in 8 cases ( 1 girl and 7 boys) on the left side. In our study the hydronephrosis which we evaluated according to SFU staging in collector system was observed mostly in left kidneys and in male cases. Conclusion: The incidence of chronic renal disease in our country is considerably high and when diagnosing is deferred it may lead to chronic renal disease. Increasing awareness and referral to appropriate center will lead to positive outcomes in long term progression of renal diseases and decreases in their prevalence.
\end{abstract}

Key Words: Renal diseases, diagnosis, USG.

${ }^{*}$ Address for Correspondence:

Dr Nazli Gulriz Ceri, MD, Assistant Professor, Adnan Menderes University Faculty of Medicine Department of Anatomy, 09010 Efeler/Aydin, TURKEY.

Email: drnazligulrizceri@gmail.com

Received Date: 04/06/2020 Revised Date: 19/07/2020 Accepted Date: 30/08/2020

DOI: https://doi.org/10.26611/10011611

This work is licensed under a Creative Commons Attribution-NonCommercial 4.0 International License. (oc)) EY-NC

\begin{tabular}{|l|l|}
\hline \multicolumn{2}{|c|}{ Access this article online } \\
\hline Quick Response Code: & Website: \\
\hline & www.medpulse.in \\
\hline & \\
\hline
\end{tabular}

\section{INTRODUCTION}

The prevalence of renal disease in children varies between $4,5-8,7 \%^{1,2}$. In early childhood, younger patients may sometimes refer to hospital with nonspecific symptoms such as refractory fever and retarded development; whereas, this problem may be completely asymptomatic in older children ${ }^{2}$. The diagnosis of lower degree hydronephrosis is frequently urinary tract infection (UTI). During later assessments or in the first year of life during imaging for non-urological causes it may emerge as prenatally ${ }^{3}$. At the same time, urinary tract dilatation or hydronephrosis which may also be called as pelvicaliectasis is widespread in children or it may be present in $1-5 \%$ of pregnancies as antenatally ${ }^{4,6}$. Therefore, to establish the prevalence of renal diseases in asymptomatic children is very difficult ${ }^{7}$. To diagnose these diseases and prevent them would be possible through increased awareness. While diagnosing hydronephrosis, staging can be made by checking the effected parts of the kidney. An ideal staging is not available yet, evaluation of hydronephrosis with USG can be made according to Society for Fetal Urology's (SFU) degree and AP diameter of pelvis ${ }^{8}$. The values obtained through measuring the AP diameter of pelvis will provide information about the severity of enlargement. In the womb this diameter is in the normal limits until $4 \mathrm{~mm}$. The measurements above this 
value are considered pathological. Postnatal measurements above $6 \mathrm{~mm}$ are also considered pathological. If the enlargement is between 7-9 $\mathrm{mm}$ it is defined as "minor" (stage 1), if between 9-15 $\mathrm{mm}$ as "medium" (stage 2), if between 16-20 $\mathrm{mm}$ as "advanced" (stage 3 ) and if over 21 $\mathrm{mm}$ as "extreme" (stage 4$)^{4}$. In hydronephrosis, according to SFU staging, the thickness of pelvis and parenchyma is normal at stage 0 . Whereas, slight dilatation is seen at stage 1 ; serious dilatation at stage 2; extreme dilatation in pelvis, enlargement at calyx and normal renal parenchyma at stage 3 ; extreme dilatation at pelvis and calyx and thinning in renal parenchyma at stage $4^{10-11}$.

\section{MATERIALS AND METHODS}

Our study was carried out between January 2015 and April 2016 with renal USG taken from 1919 patients between 015 years of age in Aydin Adnan Menderes University Hospital. Cases were evaluated in terms of gender, height, weight, body mass index (BMI), age and classified according to medical records at the clinic. Patients were also evaluated in terms of demographic parameters and hydronephrosis. In order to define demographic characteristics, cases' information related to gender, age, weight, height and BMI were included. For the diagnosis and grading of postnatal hydronephrosis, the Society of Fetal Urology (SFU) in 1993 proposed 5 different staging procedures. SFU staging examines the fullness of renal pelvis, enlargement of major and minor calyxes and cortex thickness ${ }^{4,12}$. Fig.1. According to SFU staging, hydronephrosis was divided into 5 groups; (normal hydronephrosis, G1 hydronephrosis, G2 hydronephrosis, G3 hydronephrosis and G4 hydronephrosis). For the two different grades 2 mentioned in this classification, we recorded both as grade 2. For the statistical analysis of data, the Statistical Package for the Social Sciences (SPSS 20.0) program was used. To study the relationship between two variable-groups the Mann-Whitney U test was used and in groups of variables more than one Kruskal Wallis $\mathrm{H}$ test was utilized.

\section{Statement of Ethics}

The study was approved by the Ethics Committee of the Adnan Menderes University Faculty of Medicine NonInterventional Clinical Research.

\section{RESULTS}

The mean age of all pediatric cases included in our study was $6 \pm 4.52(0-15$ years $)$ years. The mean age was calculated as $7,08 \pm 4,52$ years in female patients and 5,65 $\pm 4,41$ years in male patients. Gender distribution by age groups is shown in Table-1.

\begin{tabular}{cccc}
\multicolumn{4}{c}{ Table 1: Gender distribution by age groups } \\
\hline Age Group & Girl & Boy & Total \\
\hline 0 age group & 16 & 26 & 42 \\
1 age group & 156 & 200 & 356 \\
2 age group & 61 & 138 & 199 \\
3 age group & 43 & 64 & 107 \\
4 age group & 47 & 51 & 98 \\
5 age group & 47 & 51 & 119 \\
6 age group & 58 & 49 & 107 \\
7 age group & 57 & 48 & 105 \\
8 age group & 59 & 53 & 112 \\
9 age group & 72 & 53 & 125 \\
10 age group & 65 & 62 & 127 \\
11 age group & 55 & 41 & 96 \\
12 age group & 55 & 37 & 92 \\
13 age group & 51 & 34 & 85 \\
14 age group & 36 & 39 & 75 \\
15 age group & 48 & 26 & 74 \\
\hline
\end{tabular}

The mean, standard deviation, minimum and maximum values of weight, height and body mass indexes of pediatric cases in girls, boys and all cases are shown in the Table-2. The average BMI of all cases was found as $20,1765 \pm 5,1106 \mathrm{~kg} / \mathrm{m} 2$ (min: 9,49 kg/m2, max: 121,50 kg/m2). It was 20,1848 $\pm 4,6449 \mathrm{~kg} / \mathrm{m} 2$ (min: 11,20 kg/m2, max: 119,10 kg/m2) in girls and $20,1687 \pm 5,5118 \mathrm{~kg} / \mathrm{m} 2$ (min: $9,49 \mathrm{~kg} / \mathrm{m} 2$, max: $121,50 \mathrm{~kg} / \mathrm{m} 2$ ) in boys.

\begin{tabular}{ccccc}
\multicolumn{4}{c}{ Table 2: Statistical distribution of weight, height and body mass index } \\
\hline & & Girl & Boy & All Cases \\
\hline Weight (kg) & Mean \pm Sd & $28,56 \pm 15,97$ & $24,90 \pm 15,90$ & $26,68 \pm 16,03$ \\
& Min-max & $3,30-71,00$ & $3,15-71,00$ & $3,15-73,60$ \\
Length (cm) & Mean $\pm S d$ & $114,19 \pm 30,73$ & $106,02 \pm 31,07$ & $109,96 \pm 31,17$ \\
& Min-max & $74-170$ & $64-170$ & $64-170$ \\
BMI (kg/m ${ }^{2}$ ) & Mean $\pm S d$ & $20,18 \pm 4,64$ & $20,16 \pm 5,51$ & $20,17 \pm 5,11$ \\
\hline
\end{tabular}




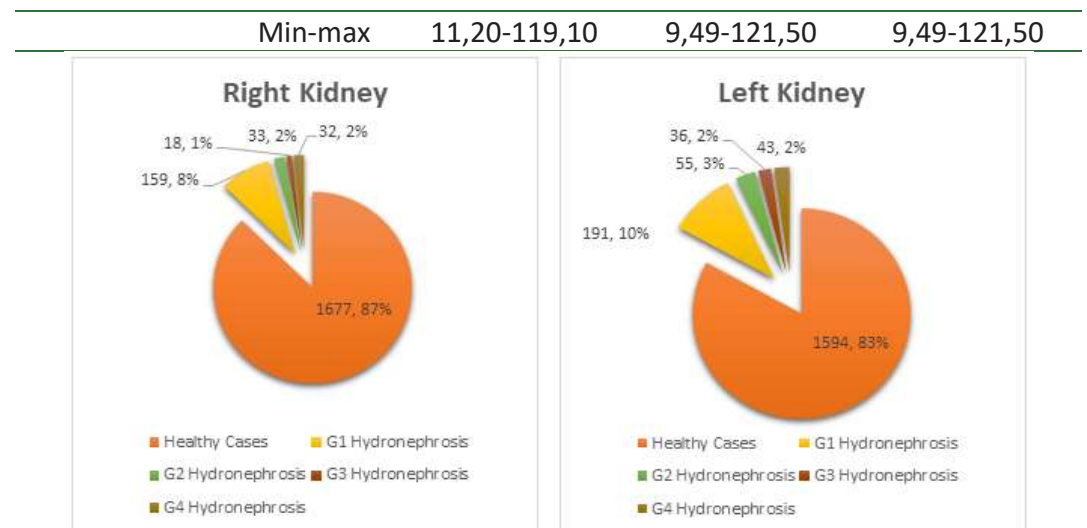

Figure 1: Distribution of Hydronephrotic Stages Determined on USG According to SFU.

Figure 1 is given by the right-left kidney hydronephrosis distribution group classification SFU. As a result of analysis performed a statistically significant difference was detected between Hydronephrosis groups seen in left kidney and age variable. $(\mathrm{p}<0,05)$ (Table 3$)$.

\begin{tabular}{|c|c|c|c|c|c|c|c|c|c|}
\hline \multicolumn{5}{|c|}{ Right renal Hydronephrosis } & \multicolumn{5}{|c|}{ Left renal Hydronephrosis } \\
\hline Age & Mean & $\begin{array}{l}\text { Std. } \\
\text { Dev, }\end{array}$ & $\begin{array}{c}\text { Kruskal-Wallis } \\
\text { H }\end{array}$ & p. & Age & Mean & $\begin{array}{l}\text { Std. } \\
\text { Dev, }\end{array}$ & $\begin{array}{c}\text { Kruskal-Wallis } \\
\text { H }\end{array}$ & p. \\
\hline 0 & 1,52 & 1,15 & 17,665 & ,281 & 0 & 1,45 &, 80 & 59,309 & ,000* \\
\hline 1 & 1,19 &, 58 & & & 1 & 1,33 & ,72 & & \\
\hline 2 & 1,16 &, 53 & & & 2 & 1,24 & 68 & & \\
\hline 3 & 1,17 &, 54 & & & 3 & 1,31 & ,82 & & \\
\hline 4 & 1,23 & ,69 & & & 4 & 1,28 & 82 & & \\
\hline 5 & 1,10 & 49 & & & 5 & 1,14 &, 49 & & \\
\hline 6 & 1,12 & ,49 & & & 6 & 1,23 & 69 & & \\
\hline 7 & 1,13 &, 54 & & & 7 & 1,21 & ,74 & & \\
\hline 8 & 1,06 & ,34 & & & 8 & 1,21 &, 58 & & \\
\hline 9 & 1,14 & ,48 & & & 9 & 1,11 & ,51 & & \\
\hline 10 & 1,10 & ,49 & & & 10 & 1,17 & ,58 & & \\
\hline 11 & 1,15 & 60 & & & 11 & 1,13 & 62 & & \\
\hline 12 & 1,18 & ,57 & & & 12 & 1,03 & ,43 & & \\
\hline 13 & 1,13 & ,40 & & & 13 & 1,11 & 41 & & \\
\hline 14 & 1,13 & ,41 & & & 14 & 1,07 & ,47 & & \\
\hline 15 & 1,12 & ,62 & & & 15 & 1,12 & 60 & & \\
\hline
\end{tabular}

As a result of analysis, a statistically significant difference could not be found between hydronephrosis group seen in left kidney and age variable, whereas there was a statistically significant difference between left hydronephrosis and gender variable This significance was observed in left kidneys and in boys (Table 4).

Table 4: In Pediatric Cases the Differences in Right-Left Kidney Hydronephrosis Group Related to Gender Variable

\begin{tabular}{cccccc}
\hline & Gender & Mean. & Std. Dev. & Mann- Whitney U & p. \\
\hline Right Kidney & GiRL & 1,13 &, 50 & 450687,000 &, 194 \\
$\begin{array}{c}\text { Hydronephrosis } \\
\text { Group }\end{array}$ & BOY & 1,18 &, 60 & & \\
Left Kidney & GiRL & 1,12 &, 47 & 416711,000 &, $000 *$ \\
Hydronephrosis & BOY & 1,30 &, 78 & &
\end{tabular}

In Right Kidney Hydronephrosis Group there was no difference in terms of weight and height but significance was seen in left kidney. Any significant difference could not be found between both kidney hydronephrosis groups and BMI (Table 5).

Table 5. In Pediatric Cases the Differences in Right-Left Kidney Hydronephrosis Group Related to Weight, Height and BMI Variables

\begin{tabular}{ccc}
\hline & Right Kidney Hydronephrosis Group & Left Kidney Hydronephrosis Group \\
& $\mathrm{P}$ & $\mathrm{p}$ \\
\hline Weight & 102 &, $000^{*}$ \\
Height &, 258 &, $000^{*}$ \\
BMI &, 205 &, 051 \\
\hline
\end{tabular}




\section{DISCUSSION}

Hydronephrosis can be defined as changes in the renal parenchyma resulting from an abnormal dilatation of the renal pelvis and calyxes. When hydronephrosis is diagnosed after birth, it is called postnatal hydronephrosis. It usually occurs when the patient presents with nonspecific symptoms after childbirth and childhood ${ }^{2,13}$. Measurement of AP diameter is used in many centers more often and the differences in the anatomy of renal pelvis may lead to misevaluation. While some radiologists measure AP diameter at the widest point, others take vertical diameter as the basis. Therefore, some writers suggest that evaluating hydronephrosis according to SFU staging not the AP diameter of pelvis would be more appropriate $^{8}$.

Killi et al.. ${ }^{14}$ have carried out this study to investigate procedures required for the surgical interventions in antenatal hydronephrosis. It was a prospective study where 44 patients with AP diameter larger than $10 \mathrm{~mm}$ were evaluated in antenatal USG. Patients were separated into two groups; follow-up group and operation group. Patients' kidney length, renal parenchyma thickness and pelvis AP diameter were scrutinized. Renal pelvis AP diameter was measured as $29,5 \pm 14,2 \mathrm{~mm}$ in surgery group and as $13,6 \pm 4,2 \mathrm{~mm}$ in follow-up group. At the end of study, a reduction was seen in renal parenchyma thickness. AP diameter of renal pelvis was selected as the most effective parameter in renal functions for surgical decision. In our study, the hydronephrosis finding that we evaluated according to SFU assessment in collector system was observed mostly in left kidney and in male cases. A significant difference was detected between age and hydronephrosis groups. In our cases G1 hydronephrosis was most prevalent $(8,3 \%)$ but G4 hydronephrosis was very few $(0,5 \%)$. Although there was a significant difference between left kidney hydronephrosis and weight and height but any significant difference could not be found with BMI. There were between groups in terms of age, gender, body weight and height, in contrast there was no significant difference with BMI. According to SFU stage $^{15}$ and pelvis AP diameter, frequency of hydronephrosis is higher in left kidney and in male cases $^{16,17}$. In our study, consistent with previous studies, hydronephrosis was observed mostly in male cases and as unilateral. Hydronephrosis was detected more frequently in male cases and left kidneys and this situation was found statistically significant. In a study, Braga et al. ${ }^{18}$ found the rates of 3-year cumulative resolution degree of 401 patients with postnatal hydronephrosis (who were graded according to Urology Association) as follows; $98 \%$ at the first degree hydronephrosis, $87 \%$ at the second degree, $76 \%$ at the third degree and $56 \%$ at the fourth degree. The 3-year resolution rates of hydronephrosis associated with postnatal urinary tract dilatation were found as; first degree (low risk) $90 \%$, second degree (medium risk) $81 \%$ and third degree (high risk) $71 \%$. Orabi et al. ${ }^{19}$ compared antenatal and the first postnatal ultrasound (US) with hydronephrosis degree. They included in their study a total of 105 cases with average gestation period of 38 weeks of them $83(79,0 \%)$ were males and $22(20,9 \%)$ were females. 105 cases first US demonstrated that $20(19,0 \%)$ had hydronephrosis; 39 (37,1\%) had slight, 29 (27,6\%) had medium and $17(16,1 \%)$ had severe hydronephrosis. Half of the hydronephrosis cases $(50,4 \%)$ showed improvement in clinic tables, whereas $(13,3 \%)$ worsened and $(36,3 \%)$ stayed same. Approximately half of all the cases (52\%) cases were diagnosed at the end of first year without showing any effect on renal function. When we evaluated the collector system in right kidney, no hydronephrotic finding could be found in 1677 (825 girls, 852 boys) cases. However, G1 hydronephrosis was observed in 159 cases ( 74 girls, 85 boys), G2 hydronephrosis in 33 cases ( 9 girls, 24 boys), G3 hydronephrosis in 18 cases ( 7 girls, 11 boys) and G4 hydronephrosis in 10 cases (4 girls, 6 boys). In our study in left kidney collector system, hydronephrosis was not seen in 1594 cases (818 girls, 776 boys). But G1 hydronephrosis was observed in 191 cases (73 girls, 118 boys), G2 hydronephrosis in 55 cases (14 girls, 41 boys), G3 hydronephrosis in 36 cases (6 girls, 30 boys) and G4 hydronephrosis in 8 cases ( 1 girl, 7 boys).

\section{CONCLUSIONS}

The incidence of chronic renal disease in our country is very high and when diagnosing is deferred it may lead to chronic renal disease. Increasing awareness and referral to appropriate center will lead to positive outcomes in long term progression of renal diseases and decreases in their prevalence. Especially chronic kidney disease is very common in our country. For this reason, it is important not to delay the diagnosis. Increasing awareness and sending patients to the appropriate centers would help getting positive results and decreasing in frequency in long-term progression of renal diseases. In our study, due to significant difference in the left kidney in terms of hydronephrosis classification and demographic features, we suggest that the clinical evaluation of the kidney and its treatment should be taken into consideration, especially in the follow-up of the left kidney in men.

\section{REFERENCES}

1. Sadeghi E. Spectrum of pediatric diseases in South Islamic Republic of Iran. Eastern Mediterranean Health Journal. 1997;103:519-29. 
2. Michael IO, Gabriel OE. Pattern of renal diseases in children in Midwestern zone of Nigeria. Saudi Journal of Kidney Diseases and Transplantation. 2003;14: 539-44.

3. Madden-Fuentes RJ, McNamara ER, Nseyo U, Wiener JS, Routh JC, Ross SS. Resolution rate of isolated low-grade hydronephrosis diagnosed within the first year of life. J Pediatr Urol, 2014;10(4):639-44.

4. Nguyen HT, Herndon CA, Cooper C, Gatti J, Kirsch A, Kokorowski P, et al.. The Society for Fetal Urology consensus statement on the evaluation and management of antenatal hydronephrosis. Journal of pediatric urology, 2010;6(3):212231.

5. Thom RP, Rosenblum ND. A translational approach to congenital non-obstructive hydronephrosis. Pediatric nephrology, 2013;28(9):1757-1761.

6. Yamaçake KG, Nguyen HT. Current management of antenatal hydronephrosis. Pediatric Nephrology. 2013;28(2):237-243.

7. Renda $R$. The evaluation of renal disease profile in children in Antalya Province. Kocaeli Medical Journal. 2017;6(2):1-6.

8. Agras K. Diagnostic evaluation of infants with antenatal hydronephrosis. Turkish Journal of Urology. 2011;37(1):4753.

9. Zwergel T, Lindenmeir T, Wullich B. Management of acute hydronephrosis in pregnancy by ureteral stenting. Eur Urol. 1996;29:292- 297.

10. Child Nephrology Association Follow-up guidelines in infants with antenatal hydronephrosis. http://cocuknefroloji.org/images/cankut-klavuz.pdf.

11. Zee RS, Herbst KW, Kim C, McKenna PH, Bentley T, Cooper $\mathrm{CS}$, et al.. Urinary tract infections in children with prenatal hydronephrosis: A risk assessment from the Society for Fetal
Urology Hydronephrosis Registry. Journal of pediatric urology. 2016;12.4:261-e1.

12. Fernbach SK, Maizels M, Conway JJ. Ultrasound grading of hydronephrosis: introduction to the system used by the Society for Fetal Urology. Pediatr Radiol. 1993;23:478.

13. Pates JA, Dashe JS. Prenatal diagnosis and management of hydronephrosis. Early human development. 2006;82.1:3-8.

14. Kıllı İ, Avlan D, Taşkınlar H, Kara PP, Apaydın FD, Delibaş A, et. al. Effective Predictors for Surgical Decision in Antenatal Hydronephrosis: A Prospective Multiparameter Analysis. Turk Journal Urologhy. 2017;43(3): 361-365.

15. Apocalypse GT, Oliveira EA, Rabelo EA, Diniz JS, Marino VS, Pereira AK, et al.. Outcome of apparent ureteropelvic junction obstruction identified by investigation of fetal hydronephrosis. Int Urol Nephrol. 2003;35:441-8.

16. Grazioli S, Parvex P, Merlini L, Combescure C, Girardin E. Antenatal and postnatal ultrasound in the evaluation of the risk of vesicoureteral reflux. Pediatr Nephrol. 2010:25:1687-1692.

17. Gürgöze MK, Karaca T. Perinatal Hydronephrosis: Etiology and Effect to Renal Functions. Firat Medical Journal. 2012;17(3):139-143.

18. Braga LH, McGrath M, Farrokhyar F, Jegatheeswaran K, Lorenzo AJ. Society for Fetal Urology Classification vs Urinary Tract Dilation Grading System for Prognostication in Prenatal Hydronephrosis: A Time to Resolution Analysis. The Journal of Urology. 2018;199(6):1615-1621.

19. Orabi M, Abozaid S, Sallout B, Shaheen AA, Heena H, Al Matary A. Outcomes of Isolated Antenatal Hydronephrosis at First Year of Life. Oman medical journal. 2018;33(2):126132.

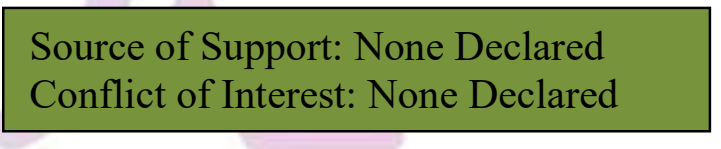

Policy for Articles with Open Access:

Authors who publish with MedPulse International Journal of Anatomy, (Print ISSN: 2348-2516) (Online ISSN: 2636-4557) agree to the following terms: Authors retain copyright and grant the journal right of first publication with the work simultaneously licensed under a Creative Commons Attribution License that allows others to share the work with an acknowledgement of the work's authorship and initial publication in this journal.

Authors are permitted and encouraged to post links to their work online (e.g., in institutional repositories or on their website) prior to and during the submission process, as it can lead to productive exchanges, as well as earlier and greater citation of published work. 